\title{
THE MONGE-AMPÈRE EQUATION AND WARPED PRODUCTS OF HIGHER RANK
}

\author{
STEFAN BECHTLUFT-SACHS ${ }^{\circledR}$ and EVANGELIA SAMIOU
}

(Received 8 April 2005; revised 6 May 2006)

Communicated by K. Wysocki

\begin{abstract}
We show that a warped product $M_{f}=\mathbb{R}^{n} \times_{f} \mathbb{R}$ has higher rank and nonpositive curvature if and only if $f$ is a convex solution of the Monge-Ampère equation. In this case we show that $M$ contains a Euclidean factor.
\end{abstract}

2000 Mathematics subject classification: primary 53C21, 53C24; secondary $35 \mathrm{~J} 60$.

\section{Introduction}

A Riemannian manifold has higher rank if along each of its geodesics there are at least two linearly independent parallel Jacobi fields. For nonpositively curved manifolds this seems to be a rather restrictive condition. An irreducible Hadamard manifold of higher rank is symmetric if it additionally admits a cocompact discontinuous group $\Gamma$ of isometries. In fact it suffices if $\Gamma$ satisfies the duality condition (see $[1,2,3,6])$. It is an open question whether this additional condition may be entirely omitted ('Rank Rigidity Conjecture').

In this note we consider warped products $M=\mathbb{R}^{n} \times f \mathbb{R}$. It turns out that nonpositive sectional curvature on $M$ translates to convexity of $f$ and that higher rank of $M$ requires $f$ to satisfy the Monge-Ampère equation. We show in Proposition 3.2 that a positive convex solution $f \in C^{\infty}\left(\mathbb{R}^{n}\right)$ of the Monge-Ampère equation has the form $f(x)=q(A x)$, where $q \in C^{\infty}\left(\mathbb{R}^{k}\right)$ is a positive convex function with $k<n$ and $A: \mathbb{R}^{n} \rightarrow \mathbb{R}^{k}$ is an orthogonal projection, that is $A A^{*}=\mathrm{id}_{\mathbb{R}^{k}}$. Hence a Riemannian manifold $M=\mathbb{R}^{n} \times{ }_{f} \mathbb{R}$ of nonpositive curvature and higher rank splits as a Riemannian product $\left(\mathbb{R}^{k} \times_{q} \mathbb{R}\right) \times \mathbb{R}^{n-k}($ Theorem 2.1).

(C) 2007 Australian Mathematical Society 1446-7887/07 \$A2.00+0.00 


\section{Warped Products of Higher Rank}

Let $M$ be a Riemannian manifold with curvature tensor $R$. The Jacobi operator $J_{v}$ of a tangent vector $v \in T_{p} M$ at $p \in M$ is the symmetric endomorphism $x \mapsto R(x, v) v$ of $T_{p} M . M$ has higher infinitesimal rank if the kernel of the Jacobi operator of each $v \in T M$ is at least 2-dimensional. By the Jacobi equation, manifolds with higher rank have this property. On the other hand, the semisymmetric spaces of conullity 2 considered in [5], [8] and [4, Theorem 2] provide examples of manifolds of rank 1 which have higher infinitesimal rank.

Given a smooth real positive function $f$ on $\mathbb{R}^{n}$ we let $M$ denote $\mathbb{R}^{n+1}=\mathbb{R}^{n} \times \mathbb{R}$ with the warped product metric given at a point $(x, t) \in M$ by

$$
g=d x^{2} \oplus f(x)^{2} d t^{2}
$$

where $d x^{2}, d t^{2}$ denote the standard metrics on $\mathbb{R}^{n}, \mathbb{R}$ respectively. We show that rank rigidity holds for these warped products.

THEOREM 2.1. The warped product $M=\mathbb{R}^{n} \times_{f} \mathbb{R}, n>0$, has nonpositive curvature and higher infinitesimal rank if and only if $f(x)=q(A x)$, where $q \in C^{\infty}\left(\mathbb{R}^{k}\right)$ is a positive convex function and $A: \mathbb{R}^{n} \rightarrow \mathbb{R}^{k}$ is an orthogonal projection with $k<n$. Thus $M$ is isometric to the product $\left(\mathbb{R}^{k} \times_{4} \mathbb{R}\right) \times \mathbb{R}^{n-k}$.

ProoF. This follows from Lemma 2.2 and Proposition 3.2.

LEMMA 2.2. $M=\mathbb{R}^{n} \times_{f} \mathbb{R}$ has nonpositive curvature and higher infinitesimal rank if and only if $f$ is a positive convex solution of the Monge-Ampère equation (3.1).

Proof. We first express the curvature tensor of $M$ in terms of $f$. To that end, let $X, Y$ and $T$ be vector fields on $M=\mathbb{R}^{n} \times_{f} \mathbb{R} \cong \mathbb{R}^{n+1}$ which are parallel with respect to the Euclidean metric on $\mathbb{R}^{n+1}$ and such that $X, Y$ are tangent to the first factor and $T=\partial / \partial t$ is tangent to the second factor. We have $g(T, T)=f^{2}$. For the covariant derivative we compute

$$
\nabla_{X} Y=0, \quad \nabla_{X} T=\nabla_{T} X=\frac{X f}{f} T \quad \text { and } \quad \nabla_{T} T=-f \operatorname{grad} f .
$$

The sectional curvature is given by

$$
\begin{aligned}
K(X+T, Y) & =\langle R(X+T, Y) Y, T+X\rangle=-\left\langle\nabla_{Y} \nabla_{T} Y, X+T\right\rangle \\
& =-\left\langle\nabla_{Y} \frac{Y f}{f} T, X+T\right\rangle=-(Y Y f) f=-f d^{2} f(Y) .
\end{aligned}
$$


For $n=1, M$ has higher infinitesimal rank if and only if $f$ is constant. Let now $n \geq 2$. By (2.1), $M$ has nonpositive curvature if and only if the Hessian $d^{2} f$ is everywhere nonnegative. For any $X$ the Jacobi operator of $X$ has at least $n$-dimensional kernel. The Jacobi operator of $X+T$ has at least 2-dimensional kernel if and only if $\langle R(X+T, Y) Y, T+X\rangle=-f d^{2} f(Y)=0$ for some $Y \neq 0$. But this is equivalent to $\operatorname{det} d^{2} f=0$.

\section{Global Convex Solutions of the Monge-Ampère Equation}

Henceforth $f: \mathbb{R}^{n} \rightarrow \mathbb{R}$ always denotes a smooth nonnegative convex solution of the Monge-Ampère equation. Thus the Hessian $d^{2} f$ of $f$ satisfies

$$
\operatorname{det} d_{x}^{2} f=0 \quad \text { and } \quad d_{x}^{2} f \geq 0
$$

for all $x \in \mathbb{R}^{n}$. On a strictly convex domain $\Omega \subset \mathbb{R}^{n}$ we have from [7] that

$$
f(x)=\sup _{L}\{L(x)\}, \quad x \in \Omega,
$$

where $L$ runs through all affine linear functions on $\mathbb{R}^{n}$ with $L \leq f$ on the boundary $\partial \Omega$.

LEMMA 3.1. For any $q \in \mathbb{R}^{n}$ with $f(q)=0$ and any $\rho>0$ we have that $q$ is contained in the convex hull of

$$
f^{-1}(0) \cap\left(q+\rho S^{n-1}\right)=\left\{z \in \mathbb{R}^{n}: f(z)=0,|z-q|=\rho\right\} .
$$

In particular there are $z_{0}, \ldots, z_{n} \in f^{-1}(0) \cap\left(q+\rho S^{n-1}\right)$ and $\lambda_{0}, \ldots, \lambda_{n} \geq 0$ with $\sum_{i=0}^{n} \lambda_{i}=1$ such that $\sum_{i=0}^{n} \lambda_{i} z_{i}=q$.

PrOOF. We assume the contrary and suppose $q=0$ and $\rho=1$, since (3.1) is invariant under translations and homotheties. The convex hull of $f^{-1}(0) \cap S^{n-1}$ is an intersection of halfspaces of $\mathbb{R}^{n}$. Hence after a suitable choice of coordinates we may further suppose that it is contained in $\left\{x_{1} \leq-2 \delta\right\}$ for some $\delta>0$. We apply (3.2) to the unit ball $\Omega=D^{n}$. Since $S^{n-1} \cap\left\{x_{1} \geq-\delta\right\}$ is compact, there is $\epsilon>0$ such that $\left.f\right|_{S^{n-1} \cap\left(x_{1} \geq-\delta\right)} \geq \epsilon$. Let $\alpha$ be the affine linear function on $\mathbb{R}^{n}$ given by

$$
\alpha\left(x_{1}, \ldots, x_{n}\right)=\left(\delta+x_{1}\right) \frac{\epsilon}{\delta+1} .
$$

Clearly $\left.\alpha\right|_{\partial \Omega} \leq\left. f\right|_{\partial \Omega}$, hence $f(0) \geq \alpha(0)=\delta \epsilon /(\delta+1)>0$, contradicting the assumption that $f(0)=0$.

PROPOSITION 3.2. Let $f \in C^{\infty}\left(\mathbb{R}^{n}\right)$ be a smooth positive function with $d_{x}^{2} f \geq 0$ and $\operatorname{det} d_{x}^{2} f=0$ for all $x \in \mathbb{R}^{n}$. Then there is a $k<n$, an orthogonal projection $A: \mathbb{R}^{n} \rightarrow \mathbb{R}^{k}$ and a $q \in C^{\infty}\left(\mathbb{R}^{k}\right)$ such that $f(x)=q(A x)$ for all $x$. 
ProOF. We may assume $f \geq 0$ and $f(0)=0$, replacing $f(x)$, if necessary, by $f(x)-f(0)-d_{0} f x$. For $\rho>0$ let $z_{i}(\rho), i=0, \ldots, n$, be as in Lemma 3.1, so that $f\left(z_{i}(\rho)\right)=0,\left|z_{i}\right|=\rho$ and $\sum_{i=0}^{n} \lambda_{i} z_{i}=0$ for some $\lambda_{i} \geq 0$ with $\sum_{i=0}^{n} \lambda_{i}=1$. Choosing a suitable sequence $\rho_{k} \rightarrow \infty$, we get convergent sequences $z_{i}\left(\rho_{k}\right) /\left|z_{i}\left(\rho_{k}\right)\right| \rightarrow$ $z_{i}(\infty)$. Then $U=\operatorname{span}\left\{z_{0}(\infty), \ldots, z_{n}(\infty)\right\}$ is a subspace of $\mathbb{R}^{n}$ of dimension $n-k>0$. We have

$$
f\left(\lambda z_{i}(\infty)\right)=\lim _{k \rightarrow \infty} f\left(\frac{\lambda z_{i}\left(\rho_{k}\right)}{\left|z_{i}\left(\rho_{k}\right)\right|}\right) \leq \lim _{k \rightarrow \infty}\left(\left(1-\frac{\lambda}{\rho_{k}}\right) f(0)+\frac{\lambda}{\rho_{k}} f\left(z_{i}\left(\rho_{k}\right)\right)\right)=0
$$

for each $\lambda \in \mathbb{R}^{+}$, since $f$ is convex and $f(0)=0$. For $\lambda \rightarrow \infty$ the convex hulls of $\left\{\lambda z_{0}(\infty), \ldots, \lambda z_{n}(\infty)\right\}$ exhaust $U$. Hence $\left.f\right|_{U}=0$.

By convexity

$$
f((1-\mu) p+u) \leq(1-\mu) f(p)+\mu f(u / \mu)=(1-\mu) f(p)
$$

for any $p \in \mathbb{R}^{n}, 0<\mu \leq 1$ and $u \in U$. In the limit $\mu \rightarrow 0$ the left hand side of (3.3) tends to $f(p+u)$, hence $f(p+u) \leq f(p)$. Since $p$ and $p+u$ are arbitrary elements of the affine space $p+U$ we conclude that $f$ is constant on the affine spaces $p+U$. We choose an isometry of the orthogonal complement $U^{\perp}$ of $U$ with $\mathbb{R}^{k}$. Let $A$ be the orthogonal projection $\mathbb{R}^{n} \rightarrow U^{\perp} \cong \mathbb{R}^{k}$ and $q$ be the restriction of $f$ to $U^{\perp} \cong \mathbb{R}^{k}$.

\section{References}

[1] W. Ballmann, 'Nonpositively curved manifolds of higher rank', Ann. of Math. 122 (1985), 597-609.

[2] W. Ballmann, M. Brin and P. Eberlein, 'Structure of manifolds of nonpositive curvature I', Ann. of Math. 122 (1985), 171-203.

[3] W. Ballmann, M. Brin and R. Spatzier, 'Structure of manifolds of nonpositive curvature II', Ann. of Math. 122 (1985), 205-235.

[4] J. Berndt and E. Samiou, 'Rank rigidity, cones and curvature-homogeneous Hadamard manifolds', Osaka J. Math. 39 (2002), 383-394.

[5] E. Boeckx, O. Kowalski and L. Vanhecke, Riemannian Manifolds of conullity two (World Scientific, Singapore, 1996).

[6] K. Burns and R. Spatzier, 'Manifolds of nonpositive curvature and their buildings', Publ. Math. Inst. Hautes Études Sci. 65 (1987), 35-59.

[7] C. E. Gutiérrez, The Monge-Ampère equation, Progr. in Nonlinear Differential Equations Appl., 44 (Birkhaüser Boston, Inc., Boston, MA, 2001).

[8] O. Kowalski, F. Tricerri and L. Vanhecke, 'Curvature-homogeneous riemannian manifolds', J. Math. Pures Appl. 71 (1992), 471-501. 
Department of Mathematics

University of Cyprus

American University of Beirut

P.O. Box 11-0236

Department of Mathematics and Statistics

Riad El Solh

P.O. Box 20537

Beirut 11072020

1678 Nicosia

Lebanon

Cyprus

e-mail: sb42.aub.edu.lb

e-mail: samiou@ucy.ac.cy 
J. Aust. Math. Soc. 83 (2007) 